\title{
Beware of Fake AGNs
}

\author{
G. Stasińska ${ }^{1}$, N. Vale Asari ${ }^{1,2}$, and R. Cid Fernandes ${ }^{2}$ \\ for the SEAGal collaboration (Semi-Empirical Analysis of GALaxies) \\ ${ }^{1}$ LUTH, Observatoire de Paris, CNRS, Université Paris Diderot; Meudon, France \\ ${ }^{2}$ Dpto. de Física - CFM - Universidade Federal de Santa Catarina, Florianópolis, SC, Brazil
}

\begin{abstract}
In the BPT diagram, the distribution of the emission-line galaxies from the Sloan Digital Sky Survey (SDSS) evokes the wings of a seagull. Traditionally, galaxies in the right wing are considered to host AGNs. Our study of the stellar populations of SDSS galaxies showed that $\sim 1 / 4$ of galaxies thought to host LINERs are in fact "retired galaxies," i.e., galaxies that stopped forming stars and are ionized by hot post-AGB stars and white dwarfs (Stasińska et al. 2008). When galaxies that lack some of the lines needed to place them in the BPT diagram are included, the fraction of retired galaxies is even larger (Cid Fernandes et al., these proceedings).
\end{abstract}

Keywords. galaxies: active, galaxies: evolution, galaxies: stellar content

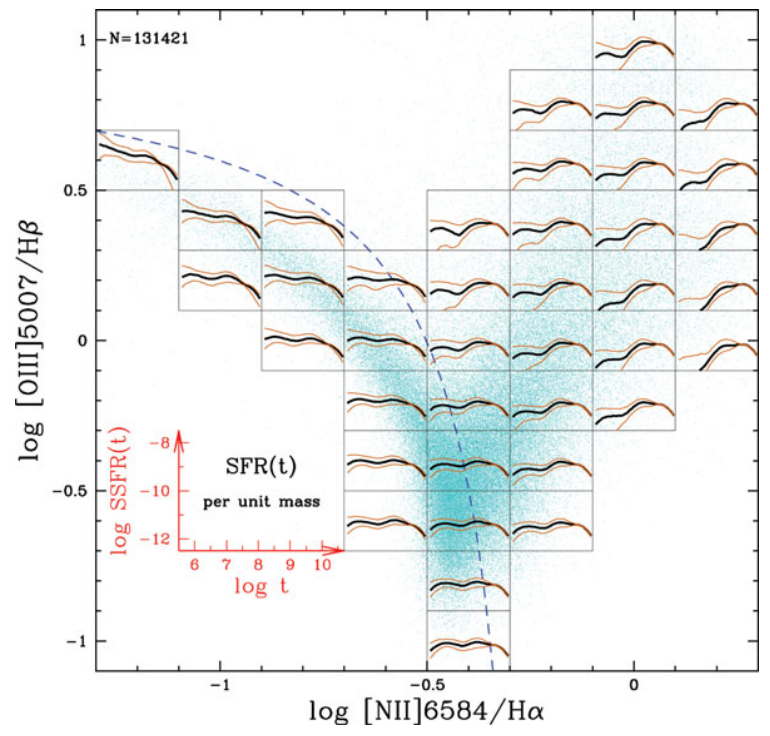

Figure 1. The BPT diagram for 131421 galaxies in the SDSS. The dashed line separates pure star forming galaxies from the rest (Stasińska et al. 2006). Superimposed are Specific Star Formation Histories obtained with the stellar population synthesis code STARLIGHT (Cid Fernandes et al. 2005). Clearly, Seyfert galaxies (upper branch of the right wing) still form stars while LINERs (lower branch) do not. Photoionization models using the radiation from the stellar populations in the LINER region are able to cover the entire BPT plane (Stasińska et al. 2008). The seagull shape is the result of selection effects.

\section{References}

Cid Fernandes, R., et al. 2005, MNRAS, 358, 363

Stasińska, G., et al. 2006, MNRAS, 371, 972

Stasińska, G., et al. 2008, MNRAS, 391, L29 